\title{
Comparison of Shear Bond Strength of Orthodontic Metal Brackets Adhered to Composite Restorations Pretreated with Er;Cr:YSGG and $\mathrm{CO}_{2}$ Lasers and Phosphoric Acid
}

\author{
Mohamad Mofateh ${ }^{1}$, Mashallah Khanemasjedi ${ }^{2} \&$ Meysam Noori $^{3}$ \\ ${ }^{1}$ Orthodontist, Ahvaz, Iran \\ ${ }^{2}$ Assistant Professor, Department of Orthodontics, School of Dental Medicine, Ahvaz Jundishapur University of \\ Medical Sciences, Ahvaz, Iran \\ ${ }^{3}$ post graduate student, Department of Orthodontics, School of Dental Medicine, Ahvaz Jundishapur University \\ of Medical Sciences, Ahvaz, Iran \\ Correspondence: Mashallah Khanemasjedi, Assistant Professor, Department of Orthodontics, School of Dental \\ Medicine, Ahvaz Jundishapur University of Medical Sciences, Ahvaz, Iran. E-mail: Masjedi_kh@yahoo.com
}

Received: December 10, 2018

Accepted: December 31, 2018 Online Published: January 29, 2019

doi:10.5539/jmbr.v8n1p14

URL: https://doi.org/10.5539/jmbr.v8n1p14

\begin{abstract}
Background \& Aim. The aim of this study was to compare the bond strength of the orthodontic brackets bonded to the composite restorations following preparations by $\mathrm{CO}_{2}$ and Er;Cr:YSGG lasers and conventional phosphoric acid etching adult orthodontic treatment. Materials \& Methods. Class V cavities were prepared on the buccal surfaces of 60 acrylic teeth and restored by composite after etching by $37 \%$ acid-etch gel. The specimens' surfaces were prepared randomly by $37 \%$ phosphoric acid etching or Er;Cr:YSGG or $\mathrm{CO}_{2}$ lasers. Central metal brackets were installed on the teeth's surfaces. The shear bond strength of the brackets to composite surfaces was measured by the crosshead speed of $1 \mathrm{~mm} / \mathrm{min}$. The scores of the remaining adhesive on the surfaces were calculated by ARI index in 5 scales. The shear bond strength values and the ARI scores were analyzed by one-way ANOVA and Chi-square tests respectively. Results. There were no significant differences among the surface preparation methods regarding bond strength between composite surfaces and the brackets. Most specimens showed ARI index of 3 in the acid phosphoric etching. In $\mathrm{CO}_{2}$ laser application, ARI index of 2 and 3 were more frequent. In Er;Cr:YSGG laser, ARI index of 3 was predominant. No significant differences existed among 3 modalities regarding scores of ARI index. Conclusion. Irradiation of $\mathrm{CO}_{2}$ and Er;Cr:YSGG lasers is recommended for clinical applications due to adequate bond strength created between the brackets and composite surfaces as well as advantages such as lower chair time and no damage to the gingival tissues.
\end{abstract}

Keywords: Surface Preparation Methods, Composite Restorations, Phosphoric Acid, Lasers of $\mathrm{CO}_{2}$ and Er;Cr:YSGG

\section{Introduction}

Currently, orthodontic treatments require a successful clinical bonding between the Orthodontic brackets and the tooth surface. In 1955, the application of a micromechanical bond between dental materials and tooth surfaces was introduced (Buonocore, 1955; Buonocore, 1981) and In the following the bond between the orthodontic brackets and enamel was introduced in 1965 (Newman, 1965). Currently, the number of adult patients requires orthodontic treatment was increased, and adult treatment has created serious and rapid issues in orthodontics (Proffit, Fields, \& Sarver, 2007). Orthodontic treatments require the ability of the bracket bands to nonenamel surfaces such as composite resins, amalgams, and porcelain. Therefore, it is important to access a reliable bond to the nonenamel surface of the tooth (Jordan, 1993). Some studies have been conducted on the strength of the orthodontic bracket to unnecessary surfaces such as amalgam or porcelain (Gross, Foley, \& Mamandras, 1997; Alavi \& Hoorfar, 2010). In the bracket bands on composite restorations, in addition to problems such as the removal of brackets, damage to dental restorations also should be considered. Therefore, some orthodontists use orthodontic bands on the teeth when facing composite restorations to do not stick brackets directly to the repairs. separation of brackets from bonded surfaces in orthodontic treatments can affect the quality of treatments, prolonged the treatment time. 
Restorative dentistry has changed a lot in recent years and the number of patients requesting beauty treatments with Mercury-Free Tooth-Colored Composite Fillings has increased (Reinhardt \& Capilouto, 1990; Burke, McHugh, Hall, Randall, Widstrom, \& Forss, 2003). Application of resin composites in the buccal level of posterior teeth, close diastema closure, Incisal surface fractures and composite veneers in the anterior teeth are recommended (Summit, Robins, \& Shwartz, 2001). Most of the composites in restorative dentistry are based on radical polymerization of methacrylates (Feilzer, De Gee, \& Davidson, 1987) in composites based on methacrylate, the chemical bond of the composite layer to the other layer involves the reaction of reactive groups of two-layer methacrylate (Vankerckhoven, Lambrechts, Van Beylen, Davidson, \& Vanherle, 1982).

These reactive methacrylate groups polymerized in the oxygen-inhibited layer composites and located on the composite surface and the layers are separately polymerized without affecting the bond strength of the next layer (Boyer \& Chan, 1984; Staxrud \& Dahl, 2011). Composites lose their surface after aging and saliva contamination (Staxrud \& Dahl, 2011; Costa, Ferreira, Klein-Júnior, Loguercio, \& Reis, 2010). the half-life of the Non-polymerized layer of the residual methacrylate groups in the composite at $37^{\circ} \mathrm{C}$ was only 50 hours. due to the problems of new composite bonding to old composite surfaces (aged), Preparation techniques for composite surfaces have been proposed to increase the strength of the orthodontic bracket bands to the composite surfaces on the tooth (Viwattanatipa, Jermwiwatkul, Chintavalakorn, \& Kanchanavasita, 2010). These techniques are divided into two categories: Mechanical and Chemical. Mechanical methods include the creation of surface roughness by the phrase, sandblast, coating with silica or by air abrasion and Chemical methods include the use of phosphoric acid, hydrofluoric acid, Silane or application of various adhesive (Gross, Foley, \& Mamandras, 1997; Bayram, Yeşilyurt, Kuşgöz, Ülker, \& Nur, 2010; Bishara, Ajlouni, \& Oonsombat, 2003). The standard protocol for the removal of the smear layer to create a successful bonding is acid-etching (Raji, Birang, Majdzade, \& Ghorbanipour, 2012). It's about two decades that Orthodontic brackets are banded using the acidetching technique (Sağır, Usumez, Ademci, \& Usumez, 2013; Yassaei, Fekrazad, Shahraki, \& Moghadam, 2014).

The etching process causes the low level of hydrophobic energy to change high levels of hydrophilic energy as well as the increased surface. the most commonly used dental surface preparation method is the use of phosphoric acid (37\%) (Sağır, Usumez, Ademci, \& Usumez, 2013). Today phosphoric acid is used for the preparation of dental surfaces and composite restorations for 15-30 seconds (Brauchli, Schramm, Senn, Ball, \& Wichelhaus, 2011).

Since the 1960s, several types of lasers have been used in medical and dental care (Raji, Birang, Majdzade, \& Ghorbanipour, 2012). In orthodontic treatments, Orbium family lasers (Er;CR:YSGG and Er:YAG) have been successfully used to prepare the enamel before the band brackets, and the irradiation of these lasers also contributes to the preparation of porcelain surface. Of course, studies on their efficacy during the preparation of direct composite restorations are limited (Sobouti, Dadgar, Sanikhaatam, Nateghian, \& Saravi, 2016).

The purpose of this study was to compare the shear bond strength of the orthodontic brackets adhered to composite restorations following preparation of Er; $\mathrm{Cr}$ :YSGG and $\mathrm{CO}_{2}$ lasers and the use of the conventional phosphoric acid method.

\section{Materials \& Methods}

This study was conducted on an experimental basis. At the buccal surface, 60 acrylic central teeth (incisors) (IdehAlMaku. Co, Tehran, Iran), class V cavities with the dimensions of $2 \times 6 \times 7 \mathrm{~mm}$ were prepared and samples were etched using 37\% etching acid gel (3M, ESPE Etching Liquid, California, USA) (Schmage, Nergiz, Herrmann, \& Özcan, 2003). The samples were restored using the restorative composite (Color A2, Unitek Z100, 3M ESPE, California, USA) based on the manufacturer's instructions and also with the use of light curing device (USA 3M Unitek, California) Ortholux LED. To carry out the aging process, all samples were stored in water at a temperature of $37^{\circ} \mathrm{C}$ for 35 days and all samples were placed in a thermocycling machine (Delta Tpo2, Nemo, Mashhad, Iran) under the thermocycling process $\left(500\right.$ cycles and $\left.55^{\circ} \mathrm{C}-5^{\circ} \mathrm{C}\right)$ (Eslamian, Borzabadi-Farahani, Mousavi, \& Ghasemi, 2011). In the next step, the samples were mounted in the acrylic blocks for better control of working conditions (Brunharo, Fernandes, de Miranda, \& Artese, 2013).

Then, samples were randomly divided into 3 groups of 20:

A) Samples of Group 1 were placed under the etching process using 37\% phosphoric acid gel for 20 seconds, washed with running water and washed with water spray and oil-free air spray and finally dried with an oil-free air spray. Metal brackets of the central tooth (incisors) (American Ortho, Sheboygan, USA) with frame in dimension of 3.32-4-48mm with bonding agent (3M, Unitek, Monrovia, California, USA) and optical composite (Transbond XT, 3M, Unitek, Monorovia, California, USA) were mounted on the teeth in such a way that their slots are parallel to the horizon and the blade of universal testing machine (K-21046, Walter+bai, Löhningen, Switzerland) is perpendicular to the body of bracket. The brackets were completely compressed on the tooth surface to maintain a minimum thickness of the composite under the bracket. Then, the lighting of the samples 
was performed by the light curing device (USA 3M Unitekt, California,) Ortholux LED from the mesial and distal side for 40 seconds.

B) All samples of Group 2 were prepared by laser Er;Cr:YSGG (Biolaser Europe GmbH, Paintweg 10, 92685 Floss, Germany) at power settings of 3 watts, the wavelength of $2780 \mathrm{~nm}$ and energy $150 \mathrm{~mJ}$ and frequency of $20 \mathrm{~Hz}$ for approximately 10 seconds (Oskoee et al., 2013). The Er;Cr:YSGG laser radiation was carried out at a distance of $1 \mathrm{~mm}$. Metal brackets of the central tooth (incisors) (American Ortho, 018 Standard. USA) with bonding agent (3M, Unitek, Monrovia, California, USA) and optical composite (Transbond XT, 3M, Unitek, Monorovia, California, USA) were mounted on the teeth in such a way that their slots are parallel to the horizon and the blade of universal testing machine (K-21046, Walter+bai, Löhningen, Switzerland) is perpendicular to the body of bracket. The brackets were completely compressed on the tooth surface to maintain a minimum thickness of the composite under the bracket. Then, the samples were lighted by the light curing device (USA 3M Unitekt, California,) Ortholux LED from the mesial and distal side for 40 seconds.

C) Samples of Group 3 were prepared by the $\mathrm{CO}_{2}$ laser (LAMBADA Scientifica Srl, Vicenza, Italy) at a power setting of 3 watts, a wavelength of $10600 \mathrm{~nm}$ and a frequency of $20 \mathrm{~Hz}$ for approximately 10 seconds. The $\mathrm{CO}_{2}$ laser radiation was performed at a focal length of $10 \mathrm{~mm}$ from the surface (the distance was determined using the hand-piece key). Metal brackets of the central tooth (incisors) (American Ortho, 018 Standard. USA) with bonding agent (3M, Unitek, Monrovia, California, USA) and optical composite resin (Transbond XT, 3M, Unitek, Monorovia, California, USA) were mounted on the teeth in such a way that their slots are parallel to the horizon and the blade of universal testing machine (K-21046, Walter+bai, Löhningen, Switzerland) is perpendicular to the body of bracket. The brackets were completely compressed on the tooth surface to maintain a minimum thickness of the composite under the bracket. Then, the samples were lighted by the light curing device (USA 3M Unitekt, California,) Ortholux LED from the mesial and distal side for 40 seconds.

Samples were stored in distilled water at a temperature of $37^{\circ} \mathrm{C}$ in an incubator for 24 hours and placed under the process of thermal cycling $\left(500\right.$ cycles at $\left.55^{\circ} \mathrm{C}-5^{\circ} \mathrm{C}\right)$ in a thermocycling machine (Delta Tpo2, Nemo, Mashhad, Iran) (Brunharo, Fernandes, de Miranda, \& Artese, 2013). A small piece of stainless steel wire $(0.017 \times 0.025$ inch $3 \mathrm{M}$ Unitek, Monrovia, California, USA) was placed in the slot bracket of all samples to minimize changes in the shape of the bracket during the debonding process and closed with an elastomeric ligature (3M Unitek, California, USA). To determine the amount of debonding forces, the universal testing machine (K-21046, Walter+bai, Löhningen, Switzerland) with a speed of $1 \mathrm{~mm} / \mathrm{min}$ was used. The samples were placed in the machine and the shear forces were applied by a lever with blade tip on the wider side of the base brackets at the site of the adhesion interface and in the occlusal-gingival direction until the bond fracture occurred. The UTM Machine calculated the force required to remove the brackets in terms of Newton, by dividing these numbers by the base bracket cross-section, the shear bond strength values were obtained in terms of MPa.

After debonding of the samples, the teeth and brackets were evaluated using a stereomicroscope (LEO 1455 VP, Germany) at 10X magnification, and the adhesive remnant index (ARI) was determined based on the amount of adhesive remnant on the surfaces according to the following criteria:

Degree 1: Remaining 100\% adhesive on the composite surface

Degree 2: Remaining more than $90 \%$ of adhesive on the composite surface

Degree 3: Remaining between $10 \%$ and $90 \%$ of adhesive on the composite surface

Degree 4: Remaining less than $10 \%$ of adhesive on the composite surface

Degree 5: No adhesive remained (Basaran, Özer, Berk, \& Hamamc1, 2007).

Finally, 2 samples from each group were randomly selected for SEM studies. For this purpose, after measuring the shear bond strength values, the samples were divided into two halves using a diamond blade and one half of them were selected to observe the contact surface. The studied samples were coated using the sputter-coated method with gold and were subjected to SEM testing and microscopic evaluation of debonding location for quality determining the enamel erosions and bonding fracture location. The shear bond strength of the brackets to the composite surfaces in the three groups by one-way ANOVA and the levels of adhesive remnant index (ARI) in them by chi-square test were analyzed.

\section{Results}

The shear bond strength of the metal brackets to the composite surfaces in the etching method with phosphoric acid was $15.42 \pm 6.33 \mathrm{MPa}$, in the $\mathrm{CO}_{2}$ laser radiation $16.32 \pm 4.93 \mathrm{MPa}$, and the shear bond strength of the metal brackets to the surfaces of composite restorations in the teeth was also estimated to be $17.01 \pm 4.87 \mathrm{MPa}$ in the Er;Cr:YSGG laser radiation (Table 1). 
Table 1. Indicators of central dispersion for shear bond strength values of metal brackets to composite surfaces in different surface preparation methods

\begin{tabular}{llllll}
\hline Preparation method & Number & Mean & SD & Minimum & Maximum \\
\hline Phosphoric acid & 20 & 15.42 & 6.33 & 4.76 & 31.23 \\
$\mathrm{CO}_{2}$ laser & 20 & 16.32 & 4.93 & 7.99 & 24.98 \\
Er;Cr:YSGG laser & 20 & 17.01 & 4.87 & 6.64 & 26.9 \\
\hline
\end{tabular}

To carry out statistical tests, first, the status of adherence or non-adherence of the data from the normal distribution was examined using Shapiro-Wilk test and the assumption of the adherence of the data to the normal distribution was confirmed. Therefore, one-way ANOVA test was used to compare the shear bond strength values of metal brackets to composite surfaces that this test did not show any significant differences between the three groups in this respect $(\mathrm{p}=0.65)$.

In examining the degrees of adhesive remnants on composite restorations and using a preparation method with the application of phosphoric acid, index 1 in 4 samples (20.0\%), index 2 in 3 samples (15.0\%), index 3 in 9 samples (45.0\%), index 4 in 3 samples (15.0\%) and index 5 in 1 sample (5.0\%) were reported, respectively. In the $\mathrm{CO}_{2}$ laser radiation, index 1 in 1 sample (5.0\%), index 2 in 9 samples (45.0\%), index 3 in 9 samples (45.0\%), index 4 in 0 samples ( $0 \%$ ) and index 5 in 1 sample (5.0\%) were reported, respectively. In the Er;Cr:YSGG laser radiation, index 1 in 0 samples ( $0 \%$ ), index 2 in 4 samples (20.0\%), index 3 in 10 samples (50.0\%), index 4 in 5 samples $(25.0 \%)$ and index 5 in 1 sample (5.0\%) were reported, respectively (Table 2$)$.

According to the results of the Chi-square test, there were no significant differences in the frequency of different degrees of ARI index in different preparation groups $(\mathrm{p}=0.08)$.

Table 2. Frequency of different degrees of adhesive remnant index (ARI) following the use of different composite surface preparation methods

\begin{tabular}{llllll}
\hline ARI index & Index 1 & Index 2 & Index 3 & Index 4 & Index 5 \\
\hline Phosphoric acid & $4(20.0 \%)$ & $3(15.0 \%)$ & $9(45.0 \%)$ & $3(15.0 \%)$ & $1(5.0 \%)$ \\
$\mathrm{CO}_{2}$ laser & $1(5.0 \%)$ & $9(45.0 \%)$ & $9(45.0 \%)$ & 0 & $1(5.0 \%)$ \\
Er;Cr:YSGG laser & 0 & $4(20.0 \%)$ & $10(50.0 \%)$ & $5(25.0 \%)$ & $1(5.0 \%)$ \\
\hline
\end{tabular}

Figures 1 to 3 show the results of evaluating samples under the Scanning Electron Microscope (SEM) device in three groups. In the phosphoric acid group (Figure 1), the prepared surface is smoother and its surface roughness is less than the other two groups. Also, according to the images, the surface roughness created on the surface of the composite restorations in the group Er;Cr:YSGG (Figure 2) is more regular and uniform than the $\mathrm{CO}_{2}$ group (Figure 3), which causes more micro-mechanical retention and less damage to the restoration surface.

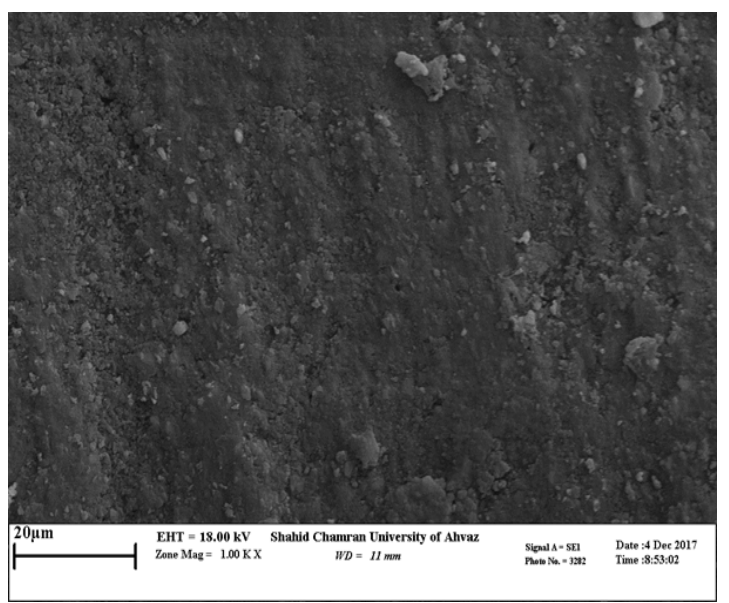

Figure 1. Image of the surface prepared with phosphoric acid under an electron microscope 


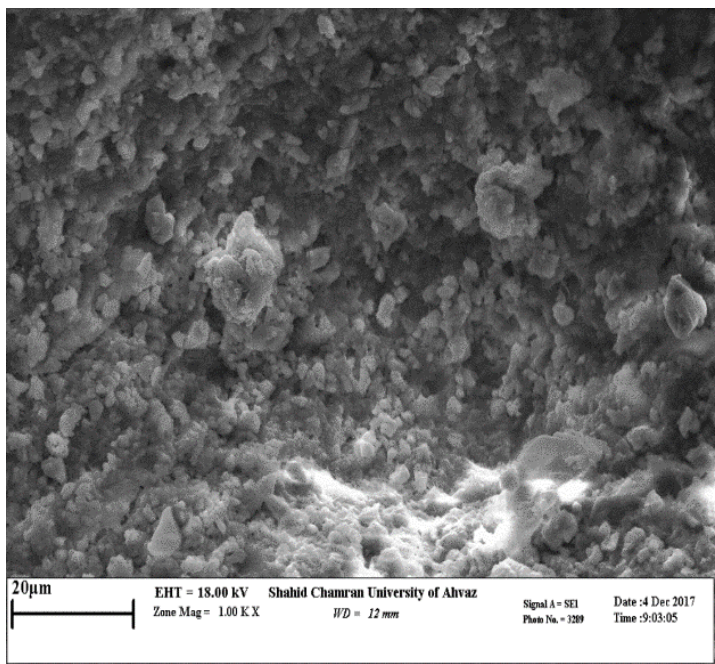

Figure 2. Image of the surface prepared with Er;Cr:YSGG laser under the electron microscope

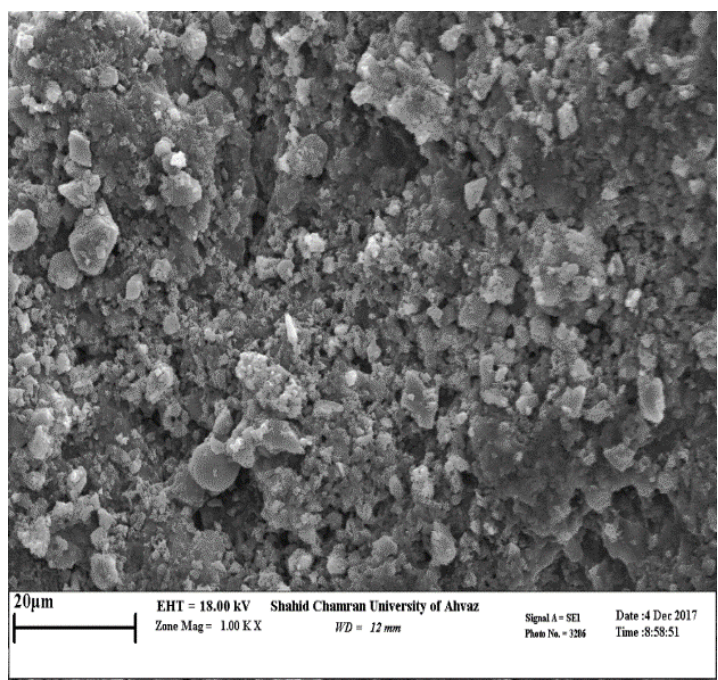

Figure 3. Image of the surface prepared with a $\mathrm{CO}_{2}$ laser under the electron microscope

\section{Discussion}

The bond strength between the orthodontic brackets and the composite restorations in the patient's teeth should be high enough to resist masticatory forces during treatment, and at the end, they should be removed without damaging in the composite surfaces.

According to the results of the present study, the mean shear bond strength of the metal orthodontic brackets to the surfaces of composite restorations following the preparation by etching methods with phosphoric acid and radiation of carbon dioxide and Er;Cr:YSGG lasers was 15.42, 16.32 and 17.01MPa, respectively, and there were no significant differences between the groups in this respect.

Different values for bond strength of brackets have been reported in the research literature. Maijer and Smith (1986) considered the bond strength of $8 \mathrm{MPa}$ for brackets to be adequate, and strength less than this value is not acceptable (Mailer \& Smith, 1986). According to the estimates of Reynolds (1975), the bond strength in bonding orthodontic brackets to enamel and restorative surfaces should be in the range of 6-8MPa (Reynolds, 1975). On the other hand, Germec et al. (2009) declared the range of acceptable bond strength for bonding brackets to be equal to 5-8MPa (Germec, Cakan, Ozdemir, Arun, \& Cakan, 2008). With regard to the above criteria, the shear bond strength values of the metal orthodontic brackets to the surfaces of composite restorations in the teeth in this study and in all three methods of using phosphoric acid and radiation of Er;Cr:YSGG and $\mathrm{CO}_{2}$ lasers were acceptable. 
In the study of Khanehmasjedi et al. (2011), there were no significant differences in the shear bond strength values of bonded orthodontic brackets to the enamel surfaces prepared using phosphoric acid and $\mathrm{CO}_{2}$ and Er;Cr:YSGG laser radiation (Leila, Alireza, \& Alireza, 2014) (Persian). In the recent study, the irradiation of Er;Cr:YSGG and $\mathrm{CO}_{2}$ lasers were performed on teeth enamel surfaces at power 3 watts $\left(8.3 \mathrm{j} / \mathrm{cm}^{3}\right)$, and the shear bond strength method was used to estimate the values of bond strength between the teeth enamel and the orthodontic brackets. The radiation power of the Er;Cr:YSGG and $\mathrm{CO}_{2}$ lasers in this study were also determined to be 3 watts that, of course, the radiations were done on the surfaces of the teeth composite restorations. Similar to the findings of the research conducted by Khanehmasjedi et al. (2011), there were no significant differences in the bond strength values of metal brackets to the surfaces of composite restorations after preparation using the procedures of phosphoric acid and $\mathrm{CO}_{2}$ and Er;Cr:YSGG lasers radiation in the present study as well. Of course, the shear bond strength of the brackets to the enamel surfaces of the teeth in the method of phosphoric acid application was more than the other methods to a limited extent in the study of Khanehmasjedi et al. (2011), but in the present study, but these results were inverse in the present study, and the Er;Cr:YSGG laser radiation produced the highest shear bond strength values (albeit insignificant) (Leila, Alireza, \& Alireza, 2014) (Persian). The surface topography of enamel and resin composite are different from each other, and therefore the bonding mechanism to them is also different. The bonding mechanism in the enamel is essentially due to mechanical retentions that keep the brackets at the surface. But chemical retentions may play a greater role in the composite resins.

Serdar et al. (2002) used the laser Er;Cr:YSGG laser with energies of 1 and 2 watts, and concluded that the radiation of this type of laser with these powers did not create sufficient bond strength during orthodontic treatments (Üşümez S., Orhan, \& Üşümez A., 2002). Therefore, in the present study, the power of 3 watts was used for each of the Er;Cr:YSGG and $\mathrm{CO}_{2}$ lasers, and the results were obtained at the level of a phosphoric acid etching method.

Alizadeh Oskoee et al. (2013) examined the effects of preparation by Er;Cr:YSGG; Nd:YAG and $\mathrm{CO}_{2}$ lasers on the shear bond strength of silorane-based repair composites, and showed that the shear bond strength of the repair composites following the preparation with lasers has been sufficient (Oskoee et al., 2013). In the recent research, the bond strength obtained in the preparation by laser radiation of Er;Cr:YSGG was significantly higher than that of the $\mathrm{CO}_{2}$ laser that the findings were not found in our study. Of course, it should be noted that study of Alizadeh Oskoee et al. (2013) was conducted on silorane-based composites that this may be one of the reasons for the difference in the results of the two studies (Oskoee et al., 2013).

Oshagh et al. (2013) evaluated the shear bond strength of orthodontic brackets in bonding and rebonding processes after preparation by $\mathrm{CO}_{2}$ laser radiation and phosphoric acid application and showed that preparation through acid etching had higher bond strength values compared to laser radiation (Oshagh, Pakshir, Najafi, Naseri, Nasrabadi, \& Torkan, 2013). Contrary to the recent research results, any significant differences between the laser groups and phosphoric acid etching were not recorded in our study. However, in recent research, laser radiation and acid etching were used for the purposes of re-bonding brackets, and bonding of brackets was also done to the teeth enamel surfaces, rather than the surfaces of composite restorations on the teeth. In general, these cases make the differences between the two investigations to be evident and clear in terms of the bond strength values of the bracket.

As well as, Kimyai et al. (2010) investigated the effects of surface preparation methods using diamond bur, air abrasion and Er;Cr:YSGG laser radiation on bond strength of laboratory repair composite resins, and indicated that Er;Cr:YSGG laser radiation has been effective in restorations of laboratory composites, similar to the air abrasion method (Kimyai, Mohammadi, Navimipour, \& Rikhtegaran, 2010). In the recent study, Gradia cylindershaped composite was used for the preparation of samples and its results were in line with our research results, although the operating protocol of the two studies was different. In a study done by Basaran et al. (2007); the shear bond strength of the orthodontic brackets following bonding to enamel through Er;Cr:YSGG laser radiation at energies of $0.5,1$ and 2 watts was examined and it was found that the average bond strength of the brackets to enamel surfaces in Er;Cr:YSGG laser radiation at powers of 1 and 2 watts was 15 seconds comparable to that of conventional acid etching (Basaran, Özer, Berk, \& Hamamc1, 2007). In the present study, the power of $3 \mathrm{~W}$ of Er;Cr:YSGG laser was used for radiation on composite restorations and results were reported as the usual method of phosphoric acid application for etching of the restorations surfaces.

On the other hand, based on the results of the present study and according to microscopic observations, the prepared surface in the phosphoric acid group was smoother and its surface roughness was lower than the other two groups. Also, the surface roughness created on the surface of the composite restorations in the samples under Er;Cr:YSGG laser radiation was more regular and uniform than those of the $\mathrm{CO}_{2}$ laser radiation that these surfaces caused more micromechanical retentions and less damage to the restoration surface. However, Yassaie et al. (2014) examined the shear bond strength of ceramic and metal brackets following preparation with 
phosphoric acid and Er:YAG laser radiation, but they did not observe any significant differences in the groups based on the results of electron microscopy (Yassaei, Fekrazad, Shahraki, \& Moghadam, 2014). This difference in results can be due to different laser radiation in two studies.

In another study recently carried out by Sobouti et al. (2016), the shear bond strength values of metal brackets to composite surfaces following the use of different surface preparation methods were investigated, and Er:YAG laser radiation at powers of 2 and 3 watts was recommended for clinical applications due to the sufficient bond strength between the brackets and the composite surfaces and the lack of problems and disadvantages of other methods (Sobouti, Dadgar, Sanikhaatam, Nateghian, \& Saravi, 2016). Although the Er:YAG laser is the previous generation of Er;Cr:YSGG laser (used in this study), both of the lasers are from the Erbium Laser family and it seems that these two lasers have good results in the preparation of surfaces of composite restorations for bonding to orthodontic brackets.

All of the above research shows the effectiveness of Erbium laser radiation for the preparation of enamel surfaces and restorations at the dental surface. Also, it seems that the morphological changes of surfaces depend on the laser energy intensity, the duration of the radiation, the distance from the radiation source to the surface, and the flow of water (Rodríguez-Vilchis, Contreras-Bulnes, Sánchez-Flores, \& Samano, 2010). Due to the creation of sufficient bond strength in the radiation of Er;Cr:YSGG and $\mathrm{CO}_{2}$ lasers on composite restorations in the present study, it seems that there is no need for surface roughness on composites using sandblasting methods with aluminum particles or the use of diamond bur, and also the risk of gingival damage following the application of acid etching is also eliminated. On the other hand, the preparation time in laser radiation is significantly reduced in comparison with the use of acid etching with acid phosphoric (about 10 seconds versus 2 minutes) that the finding is very desirable in clinical conditions for the patient and clinician (Hosseini, Sobouti, Etemadi, \& Chiniforush, 2015). Brauchli et al. (2011) examined the results of bovine enamel preparation through Er:YAG and $\mathrm{CO}_{2}$ lasers radiation in the status of surface structure and shear bond strengths, and demonstrated that the conventional acid etching method had a higher bond strength compared with the two laser groups (Brauchli, Schramm, Senn, Ball, \& Wichelhaus, 2011). In this study, the lack of significant difference between the laser groups with high standard deviation values of the shear bond strength of the brackets in them was attributed. Based on statistical relations, the significance of the results decreases with increasing the standard deviation values of a group of data. The difference between the results of this study and the results of the study by Brachli et al. (2011) is probably because of the difference in the type of substrate. On the other hand, Tehranchi et al. (2011) investigated the effects of $\mathrm{CO}_{2}$ laser radiation in debonding of ceramic brackets, and represented that $\mathrm{CO}_{2}$ laser radiation can replace conventional ceramic bracket bonding methods to dental surfaces (Tehranchi, Fekrazad, Zafar, Eslami, Kalhori, \& Gutknecht, 2011) that the findings are consistent with the results of the present study, although different brackets in two studies have been evaluated.

Due to the lack of report of significant differences in terms of acid etching time in different studies, the time of 20 seconds acid etching was considered in this study. Of course, the total etching times were not recorded in the current study, but it seems that the laser radiation leads to saving time, because, unlike the acid etching process in the use of lasers, there is no need to wash the surface of samples after etching. The approximate time for tooth cleaning is about 15 to 30 seconds, and therefore, the time required for complete bonding of the teeth in the mouth is about 300 to 600 seconds. It seems that saving 5 to 10 minutes for patient treatment by immediate implantation of brackets in teeth under laser radiation is an important issue in this regard. Of course, there is a need for a lot of research to determine the exact time of clinical work on this respect. According to the results of this study, there were no significant differences in terms of the frequency of ARI index values among different groups. In the preparation through the application of phosphoric acid, most of the samples (45.0\%) had an index ARI 3 and the lowest (5.0\%) had an index ARI 5. Most samples (45.0\%) had ARI indexes 2 and 3 in $\mathrm{CO}_{2}$ laser radiation, and at the same time, indexes 1 and 5 had minimal ARI index values. On the other hand, the index ARI 3 was reported for about half of the samples in Er;Cr:YSGG laser radiation. The least frequency was related to the index ARI 5. In research conducted by Hosseini et al. (2012), there were no significant differences in the frequency of ARI indices in laser and acid etching groups, which is consistent with the present study in this regard (Hosseini et al., 2012). However, Gokcelik et al. (2007) observed higher ARI degrees in the samples under Er-YAG laser radiation compared to the acid etching group (Gokcelik, 2007). Also, Alavi et al. (2014) compared the results of brackets bonding to enamel following acid etching and Er:YAG laser radiation, and reported that there were no significant differences in the ARI index in the groups (Alavi, Birang, \& Hajizadeh, 2014). Although the study by Alavi et al. (2014) on the enamel substrates of the teeth and the present study on the substrates of composite restorations on them have been done, the results obtained from the ARI index are not consistent with the two studies.

In the study carried out by Tehranchi et al. (2011), significant differences between the groups of non-radiation of laser and super pulse $\mathrm{CO}_{2}$ laser radiation in the ARI index during the study of effects of $\mathrm{CO}_{2}$ laser radiation in 
debonding of ceramic brackets that the finding was contrary to the results of the present study (Tehranchi, Fekrazad, Zafar, Eslami, Kalhori, \& Gutknecht, 2011).

Different types of brackets, as well as different laser radiation parameters, can be the reasons related to differences in these results. Also, in a study conducted by Basaran et al. (2007), there were no significant differences in the values of adhesive remnants followed by debonding of orthodontic brackets in etching techniques with Er;Cr:YSGG laser at energies of 0.5, 1, and 2 watts (Basaran, Özer, Berk, \& Hamamc1, 2007). This was also documented in the present study, although the Er;Cr:YSGG laser with a power of 3 watts was used in it.

The greater frequency of index ARI 3 in the present study indicates that the greater debonding in the resin-tooth contact surfaces occurred in the surface preparation methods of composite restorations. This type of debonding is clinically more desirable, because the need to clean the debonded enamel (restoration surfaces) is decreased in this case and, consequently, the risk of damage to the enamel or restoration will be reduced (Sinha, Nanda, Duncanson, \& Hosier, 1995). The frequency of this type of debonding in clinical conditions is more, because providing a favorable etching on the tooth surface is more difficult in this case, due to the lack of control of the humidity, temperature, time and patient movements (Fernandez \& Canut, 1999).

The lowest frequency of ARI indexes in the present study was related to indexes 4 and 5. Indexes 4 and 5 show that the debonding occurred in the contact surface of the tooth and the resin, and the need to clean the debonded area was also less and, at the same time, the risk of enamel erosion or the surfaces of composite restorations on it was low. Of course, it is better that debonding would be occurred in the contact surface of the bracket-resin or inside the resin (Sinha, Nanda, Duncanson, \& Hosier, 1995), because the stresses affecting the enamel surfaces will be greater if the less adhesive remains on the teeth (Hashem-Hoseini et al., 2008). In addition, due to the base structure of the brackets, the debonding is in the contact surface of the resin and the bracket is uncommon (Smith \& Maijer, 1983). However, Lee et al. (2003) reported more fracture patterns in the contact surface of bracket-resin (Lee et al., 2003) when evaluating bonded brackets following Er:YAG laser irradiation or acid etching. These differences can be related to the type of bond strength test that it was the tensile bond strength in the study of Lee et al. (2003) and the shear bond strength in this study. At present, the ideal pattern of bond fracture between metal brackets and resin composites has not been specified, although metal brackets are often fractured at the base of the resin-bracket (Joseph \& Rossouw, 1990).

\section{Conclusion}

Therefore, all of the surface preparation methods used to provide sufficient bond strength between the metal brackets and the surfaces of composite restorations, and radiation of Er;Cr:YSGG and $\mathrm{CO}_{2}$ lasers were recommended because of the creation of sufficient bond strength between the brackets and the surfaces of composite restorations and advantages such as the lack of damage to the gingival tissues and less operating time for clinical applications.

\section{Conflict of interests}

The authors declare that there is no conflict of interests regarding the publication of this paper.

\section{References}

Alavi, S., \& Hoorfar, A. (2010). Evaluation of porcelain preparation procedures on shear bond strength of steel brackets. Majallah i Dandanpizishki (Journal of Islamic Dental Association of Iran, 22(1).

Alavi, S., Birang, R., \& Hajizadeh, F. (2014). Shear bond strength of orthodontic brackets after acid-etched and erbium-doped yttrium aluminum garnet laser-etched. Dental Research Journal, 11(3), 321.

Basaran, G., Özer, T., Berk, N., \& Hamamc1, O. (2007). Etching enamel for orthodontics with an erbium, chromium: Yttrium-scandium-gallium-garnet laser system. The Angle Orthodontist, 77(1), 117-24.

Bayram, M., Yeşilyurt, C., Kuşgöz, A., Ülker, M., \& Nur, M. (2010). Shear bond strength of orthodontic brackets to aged resin composite surfaces: Effect of surface conditioning. The European Journal of Orthodontics, 33(2), 174-9.

Bishara, S. E., Ajlouni, R., \& Oonsombat, C. (2003). Bonding Orthodontic Brackets to Composite Using Different Surface Preparations and Adhesive/Primers: A Comparative Study. World Journal of Orthodontics, 4(4).

Boyer, D., Chan, K. C., \& Reinhardt, J. (1984). Build-up and repair of light-cured composites: Bond strength. Journal of Dental Research, 63(10), 1241-4.

Brauchli, L. M., Schramm, A., Senn, C., Ball, J., \& Wichelhaus, A. (2011). Laser conditioning of enamel with the Erbium YAG and the $\mathrm{CO}_{2}$ laser. Bond strength and surface structure. Dentistry, 1(2), 1-5. 
Brunharo, I. H., Fernandes, D. J., de Miranda, M. S., \& Artese, F. (2013). Influence of surface treatment on shear bond strength of orthodontic brackets. Dental Press Journal of Orthodontics, 18(3), 54-62.

Buonocore, M. G. (1955). A simple method of increasing the adhesion of acrylic filling materials to enamel surfaces. Journal of Dental Research, 34(6), 849-53.

Buonocore, M. G. (1981). Retrospections on bonding. Dental Clinics of North America, 25(2), 241-55.

Burke, F., McHugh, S., Hall, A., Randall, R., Widstrom, E., \& Forss, H. (2003). Amalgam and composite use in UK general dental practice in 2001. British Dental Journal, 194(11), 613.

Burtscher, P. (1993). Stability of radicals in cured composite materials. Dental Materials, 9(4), 218-21.

Costa, T., Ferreira, S., Klein-Júnior, C., Loguercio, A., \& Reis, A. (2010). Durability of surface treatments and intermediate agents used for repair of a polished composite. Operative Dentistry, 35(2), 231-7.

Eslamian, L., Borzabadi-Farahani, A., Mousavi, N., \& Ghasemi, A. (2011). The effects of various surface treatments on the shear bond strengths of stainless steel brackets to artificially-aged composite restorations. Australian Orthodontic Journal, 27(1), 28.

Feilzer, A., De Gee, A., \& Davidson, C. (1987). Setting stress in composite resin in relation to configuration of the restoration. Journal of Dental Research, 66(11), 1636-9.

Fernandez, L., \& Canut, J. A. (1999). In vitro comparison of the retention capacity of new aesthetic brackets. The European Journal of Orthodontics, 21(1), 71-7.

Germec, D., Cakan, U., Ozdemir, F. I., Arun, T., \& Cakan, M. (2008). Shear bond strength of brackets bonded to amalgam with different intermediate resins and adhesives. The European Journal of Orthodontics, 31(2), 207-12.

Gokcelik et al. (2007). The influence of Er:YAG laser conditioning versus self-etching adhesives with acid etching on the shear bond strength of orthodontic brackets. Photomedicine and Laser Surgery, 25(6), 50812.

Gross, M. W., Foley, T. F., \& Mamandras, A. H. (1997). Direct bonding to Adlloy-treated amalgam. American Journal of Orthodontics and Dentofacial Orthopedics, 112(3), 252-8.

Hashem-Hoseini et al. (2008). Effect of fast curing lights, argon laser, and plasma arc on bond strengths of orthodontic brackets: An in vitro study. Journal of Dentistry of Tehran University of Medical Sciences, 5(4), 167-72.

Hosseini et al. (2012). Comparison of shear bond strength of orthodontic brackets bonded to enamel prepared by Er:YAG laser and conventional acid-etching. Journal of Dentistry (Tehran, Iran), 9(1), 20.

Hosseini, M. H., Sobouti, F., Etemadi, A., \& Chiniforush, N. (2015). Shear bond strength of metal brackets to feldspathic porcelain treated by Nd:YAG laser and hydrofluoric acid. Lasers in Medical Science, 30(2), $837-41$.

Jordan, R. E. (1993). Resin to resin bonding. Esthetic Composite Bonding 2nd Ed. St Louis, MO: Mosby Year Book, 339-47.

Joseph, V., \& Rossouw, E. (1990). The shear bond strengths of stainless steel and ceramic brackets used with chemically and light-activated composite resins. American Journal of Orthodontics and Dentofacial Orthopedics, 97(2), 121-5.

Kimyai, S., Mohammadi, N., Navimipour, E. J., \& Rikhtegaran, S. (2010). Comparison of the effect of three mechanical surface treatments on the repair bond strength of a laboratory composite. Photomedicine and Laser Surgery, 28(S2), S-25-S-30.

Lee et al. (2003). Bond strengths of orthodontic bracket after acid-etched, Er:YAG laser-irradiated and combined treatment on enamel surface. The Angle Orthodontist, 73(5), 565-70.

Leila, K. M. M. B., Alireza, R., \& Alireza, R. (2014). Comparative study of shear bond strength of Orthodontic brackets adhered to the enamel of preparation with three methods of $\mathrm{CO}_{2}$ laser and Er;Cr:YSSG Hydrokinetic and phosphoric acid. Journal of Jundishapur Medical Sciences, 13(4), 425-419.

Mailer, R., \& Smith, D. (1986). Crystal growth on the outer enamel surface-an alternative to acid etching. American Journal of Orthodontics and Dentofacial Orthopedics, 89(3), 183-93.

Newman, G. V. (1965). Epoxy adhesives for orthodontic attachments: Progress report. American Journal of Orthodontics and Dentofacial Orthopedics, 51(12), 901-12. 
Oshagh, M., Pakshir, H. R., Najafi, H. Z., Naseri, M. M., Nasrabadi, N. I., \& Torkan, S. (2013). Comparison of the shear bond strength of orthodontic brackets in bonding and rebonding: Preparation with laser versus conventional acid etch technique. Photomedicine and Laser Surgery, 31(8), 360-4.

Oskoee et al. (2013). Effect of surface treatment with Er;Cr:YSSG, Nd:YAG, and $\mathrm{CO}_{2}$ lasers on repair shear bond strength of a silorane-based composite resin. Journal of Dental Research, Dental Clinics, Dental Prospects, 7(2), 61.

Proffit, W., \& Fields, H. (2007). Sarver DMContemporary Orthodontics 4th Edition Mosby. Inc, St Louis., 167-8.

Raji, S. H., Birang, R., Majdzade, F., \& Ghorbanipour, R. (2012). Evaluation of shear bond strength of orthodontic brackets bonded with Er-YAG laser etching. Dental Research Journal, 9(3), 288.

Reinhardt, J. W., \& Capilouto, M. L. (1990). Composite resin esthetic dentistry survey in New England. The Journal of the American Dental Association, 120(5), 541-4.

Reynolds, I. (1975). A review of direct orthodontic bonding. British Journal of Orthodontics, 2(3), 171-8.

Rodríguez-Vilchis, L. E., Contreras-Bulnes, R., Sánchez-Flores, I., \& Samano, E. C. (2010). Acid resistance and structural changes of human dental enamel treated with Er:YAG laser. Photomedicine and Laser Surgery, 28(2), 207-11.

Sağır, S., Usumez, A., Ademci, E., \& Usumez, S. (2013). Effect of enamel laser irradiation at different pulse settings on shear bond strength of orthodontic brackets. The Angle Orthodontist, 83(6), 973-80.

Schmage, P., Nergiz, I., Herrmann, W., \& Özcan, M. (2003). Influence of various surface-conditioning methods on the bond strength of metal brackets to ceramic surfaces. American Journal of Orthodontics and Dentofacial Orthopedics, 123(5), 540-6.

Sinha, P. K., Nanda, R. S., Duncanson, M. G., \& Hosier, M. J. (1995). Bond strengths and remnant adhesive resin on debonding for orthodontic bonding techniques. American Journal of Orthodontics and Dentofacial Orthopedics, 108(3), 302-7.

Smith, D. C., \& Maijer, R. (1983). Improvements in bracket base design. American Journal of Orthodontics, 83(4), 277-81.

Sobouti, F., Dadgar, S., Sanikhaatam, Z., Nateghian, N., \& Saravi, M. G. (2016). Effects of two erbium-doped yttrium aluminum garnet lasers and conventional treatments as composite surface abrasives on the shear bond strength of metal brackets bonded to composite resins. Journal of Orthodontic Science, 5(1), 18.

Staxrud, F., \& Dahl, J. E. (2011). Role of bonding agents in the repair of composite resin restorations. European Journal of Oral Sciences, 119(4), 316-22.

Summit, J., Robins, J., \& Shwartz, R. (2001). Fundamentals of operative dentistry (2nd ed.). Chicago: Quintessence.

Tehranchi, A., Fekrazad, R., Zafar, M., Eslami, B., Kalhori, K. A., \& Gutknecht, N. (2011). Evaluation of the effects of $\mathrm{CO}_{2}$ laser on debonding of orthodontics porcelain brackets vs. the conventional method. Lasers in Medical Science, 26(5), 563-7.

Üşümez, S., Orhan, M., \& Üşümez, A. (2002). Laser etching of enamel for direct bonding with an Er;Cr:YSSG hydrokinetic laser system. American Journal of Orthodontics and Dentofacial Orthopedics, 122(6), 649-56.

Vankerckhoven, H., Lambrechts, P., Van Beylen, M., Davidson, C., \& Vanherle, G. (1982). Unreacted methacrylate groups on the surfaces of composite resins. Journal of Dental Research, 61(6), 791-6.

Viwattanatipa, N., Jermwiwatkul, W., Chintavalakorn, R., \& Kanchanavasita, W. (2010). Weibull analysis of bond strength of orthodontic buccal tubes bonded to resin composite surface with various techniques. Orthodontic Waves, 69(2), 66-74.

Yassaei, S., Fekrazad, R., Shahraki, N., \& Moghadam, M. G. (2014). A comparison of shear bond strengths of metal and ceramic brackets using conventional acid etching technique and Er:YAG laser etching. Journal of Dental Research, Dental Clinics, Dental Prospects, 8(1), 27.

\section{Copyrights}

Copyright for this article is retained by the author(s), with first publication rights granted to the journal.

This is an open-access article distributed under the terms and conditions of the Creative Commons Attribution license (http://creativecommons.org/licenses/by/4.0/). 